\title{
DRUG-INDUCED LIVER INJURY AS A DIFFERENTIAL DIAGNOSIS OF HEPATIC MANIFESTATIONS ASSOCIATED WITH SYSTEMIC LUPUS ERYTHEMATOSUS
}

Marília Bulhões Calheiros ${ }^{1, \star}$, Alisson Aliel Vigano Pugliesi ${ }^{1}$, Walace Magalhães Barbosa ${ }^{1}$, Marcelo Gustavo Lopes ${ }^{1}$, Mariana Frason Fernandes ${ }^{1}$, Marlone Cunha da Silva ${ }^{1}$, Simone Appenzeller ${ }^{1}$, Lilian Tereza Costallat ${ }^{1}$, Lais Naziozeno Santos ${ }^{1}$

1.Universidade Estadual de Campinas, Campinas (SP), Brazil.

*Corresponding author: lilabulhoesc@gmail.com

\section{BACKGROUND}

Hepatic manifestations attributed to systemic lupus erythematosus (SLE) activity are uncommon, which makes the management of patients with SLE and liver injury a challenge. The wide range of differential diagnoses includes: overlap with another primary autoimmune liver disease, drug-induced liver injury (DILI), viral hepatitis and thrombotic liver diseases. Below, an illustrative case of a patient who - despite evidence of SLE activity - presented with liver damage secondary to a drug.

\section{CASE REPORT}

A 20-year-old female patient, diagnosed with SLE 2 years ago with the following criteria: antinuclear antibody (ANA) positive 1/160 nuclear dense fine speckled, anti-DNA positive, complement consumption, malar rash, arthritis. She had been using only hydroxychloroquine since the diagnosis. It was started azathioprine $100 \mathrm{mg} / \mathrm{day}$ (reason not clear), progressing with daily fever, jaundice, choluria and fecal acholia after using the drug for 3 weeks. Patient was hospitalized in a tertiary center for clinical investigation. Laboratory tests showed bilirubin $15 \mathrm{mg} / \mathrm{dL}$ with a predominance of direct bilirubin, a significant increase in canalicular enzymes and increase of $3 \times$ the higher value of transaminase reference. Viral hepatitis serologies were negative, as well as markers of immunemediated liver disease. Cholestatic syndrome was questioned as SLE activity since the patient had also anti-DNA positive 1/320, complement consumption of C3 and C4 and daily fever, no other noteworthy changes. However, a liver biopsy was performed with morphological findings favoring a toxic drug effect. In view of the temporal relationship between symptoms and the onset of azathioprine, the latter was considered responsible for hepatotoxicity. The patient was discharged using ursodeoxycholic acid with complete resolution of liver injury after 9 months of azathioprine suspension.

\section{CONCLUSION}

Rheumatologists should always consider the possibility of an alternative diagnoses before attributing liver injury to SLE, given its rarity. Drug-induced liver injury is always a differential diagnosis since several drugs used in the treatment of SLE have hepatotoxic potential. 